\title{
KNUT CONSEMÜLLER zum 80er
}

\author{
Bruno Hribernik ${ }^{1}$, Michael Binder ${ }^{2}$ und Gerhard Hackl ${ }^{1}$
}

${ }^{1} \mathrm{AMSET}$, Leoben, Österreich

${ }^{2}$ FFG (Österreichische Forschungsförderungs $\mathrm{GmbH}$ ), Wien, Österreich

Angenommen 8. Juni 2021; online publiziert 1. Juli 2021

Am 22.05.2021 feierte Dipl.-Ing. Dipl.-Wirtschafts-Ing. Dr.Ing. Dr.h.c. Knut Consemüller (Abb. 1) seinen achtzigsten Geburtstag. Sein beruflicher Werdegang war maßgeblich vom Werkstoff Stahl und Metallurgie beeinflusst.

Knut Consemüller absolvierte sein Studium der Eisenhüttenkunde an der Rheinisch-Westfälischen-Technischen Hochschule (RWTH) Aachen und ein wirtschaftswissenschaftliches Aufbaustudium an der RWTH Aachen und der Universität Köln und schloss seine Studien an der RWTH Aachen mit der Promotion im Jahr 1969 ab.

Von 1969 bis 1989 war Knut Consemüller im HOESCH Konzern (später ESTEL HOESCH HOOGOVENS NV) beschäftigt und war von 1980 bis 1989 als technischer Vorstand der ESTEL HOESCH HÜTTENWERKE AG in Dortmund und Nimwegen tätig.

Knut Consemüller war in dieser Zeit Vorstandsmitglied des Vereins Deutscher Eisenhüttenleute (VDEh) und Mitglied im Technologie-Beirat des Bundesministers für Forschung und Technologie in Bonn.

Im Jahr 1989 wechselte Knut Consemüller zur VOEST ALPINE STAHL AG in Linz als Direktor für Strategie- und Technologieplanung. Im Jahr 1991 wurde er zum Vorstandsmitglied der Böhler-Uddeholm AG bestellt. Dabei gestaltete er maßgeblich den Wandel vom Edelstahlhersteller zum Werkstoffkonzern, heute High Perfomance Metal Division der voestalpine AG, mit.

Von 2001-2005 war Knut Consemüller Vorsitzender der Eisenhütte Österreich/ASMET. Als Vorsitzender des Rats für Forschung und Technologieentwicklung der österreichischen Bundesregierung trug er von 2000 bis 2010 maßgeblich zur Weiterentwicklung der österreichischen Forschungs- und Technologiepolitik bei. In dieser Zeit schloss Österreich zur Spitzengruppe der EU Staaten gemäß EU Score Board auf.

\section{B. Hribernik ( $\triangle)$}

AMSET,

Franz-Josef-Straße 18,

A-8700 Leoben, Österreich

Bruno.Hribernik@gmail.com
Von 2006 bis 2011 war Knut Consemüller stellvertretender Vorsitzender des Forschungsrates der Steiermärkischen Landesregierung.

In seinen Funktionen gab er zahlreiche entscheidende Impulse zur Innovation und Wettbewerbsfähigkeit. Er förderte den Aufbau von Kompetenzzentren in Österreich und initiierte das K2 Zentrum MCL - Materials Center Leoben Forschung $\mathrm{GmbH}$, heute eine international anerkannte Forschungs- und Entwicklungsinstitution mit fast 200 ForscherInnen und TechnikerInnen, in der er Mitglied des Aufsichtsrats ist.

Knut Consemüller erhielt folgende Ehrungen und Auszeichnungen:

- Österreichisches Ehrenkreuz für Wissenschaft und Kunst 1. Klasse

- Ehrensenator der Montanuniversität Leoben

- Doktor Der Technischen Wissenschaften Ehrenhalber der TU Graz

Besonders hervorgehoben werden soll an dieser Stelle die Rolle von Knut Consemüller bei der Neuausrichtung der Eisenhütte Österreich, d. h. Gründung der ASMET vor zwan-

Abb. 1: Knut Consemüller

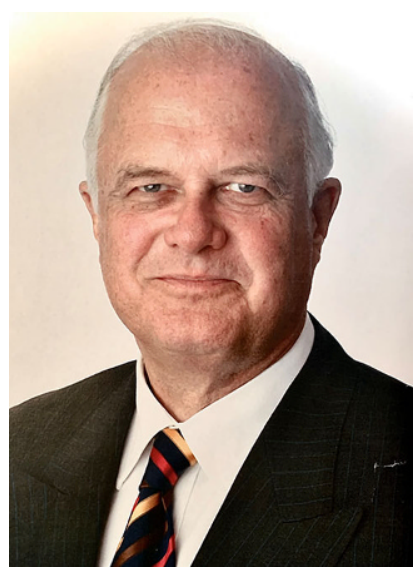


zig Jahren. Er hat dabei nicht nur in seiner damaligen Funktion als Präsident der ASMET mitgewirkt, sondern auch als Forschungs- und Technikvorstand der Böhler Uddeholm AG und durch seine guten Kontakte zur Industrie wesentlich dazu beigetragen, die Wege zu ebnen und die Mittel für die Startphase der ASMET einzuwerben. Die Gründung ASMET war gleichzeitig auch eine Initialzündung für die strategischen Kooperationen wichtiger Firmen im Bereich der Stahl-, Metallherstellung und -verarbeitung, dem Metallurgieanlagenbau sowie deren Zulieferanten in Österreich. Dies bedeute einen entscheidenden Schritt zur Erhöhung der internationalen Sichtbarkeit der österreichischen Industrie und insbesondere der Forschung am Sektor Material und Metallurgie.

Seit 2009 ist Knut Consemüller Aufsichtsrat und anschließend Vorsitzender des Beirats der Fraunhofer Austria Research $\mathrm{GmbH}$.
Knut Consemüller ist sicherlich ein Musterbeispiel für aktives Altern, denn ein Ende seiner Aktivitäten zeichnet sich nicht ab. Mit ungebrochenem Enthusiasmus wirkt er als Ratgeber und Förderer für Forschung und Innovation.

Er ist seit 1971 mit Jutta, geb. Zernecke, verheiratet. Die Familie lebt mit Kindern, Schwieger- und Enkelkindern in Wien.

Wir wünschen Dir, lieber Knut, noch viele schöne Jahre und vor allem Gesundheit.

Glückauf!

Bruno Hribernik, Michael Binder, Gerhard Hackl 